\title{
Time evolution of a superposition of dressed oscillator states in a cavity
}

\author{
G Flores-Hidalgo ${ }^{1}$, C A Linhares ${ }^{2}$, A P C Malbouisson ${ }^{3}$ and J \\ M C Malbouisson ${ }^{4}$ \\ ${ }^{1}$ Instituto de Ciências Exatas, Universidade Federal de Itajubá, 37500-903, Itajubá, \\ MG, Brazil \\ ${ }^{2}$ Instituto de Física, Universidade do Estado do Rio de Janeiro, 20559-900, Rio de \\ Janeiro, RJ, Brazil \\ ${ }^{3}$ Centro Brasileiro de Pesquisas Físicas/MCT, 22290-180, Rio de Janeiro, RJ, Brazil \\ ${ }^{4}$ Instituto de Física, Universidade Federal da Bahia, 40210-340, Salvador, BA, Brazil \\ E-mail: gfloreshidalgo@unifei.edu.br, linharescesar@gmail.com, \\ adolfo@cbpf .br,jmalboui@ufba.br
}

\begin{abstract}
Using the formalism of renormalized coordinates and dressed states introduced in previous publications, we perform a nonperturbative study of the time evolution of a superposition of two states, the ground state and the first excited level of a harmonic oscillator, the system being confined in a perfectly reflecting cavity of radius $R$. For $R \rightarrow \infty$, we find dissipation with dominance of the interference terms of the density matrix, in both weak- and strong-coupling regimes. For small values of $R$ all elements of the density matrix present an oscillatory behavior as times goes on and the system is not dissipative. In both cases, we obtain improved theoretical results with respect to those coming from perturbation theory.
\end{abstract}

PACS numbers: 03.65.Ca,03.65.Yz

Submitted to: J. Phys. A: Math. Gen. 


\section{Introduction}

The main analytical method used to treat the physics of interacting systems is perturbation theory. In this framework, bare, noninteracting, matter fields and gauge fields (to which bare quanta are associated) are introduced, the interaction being accounted for order by order in powers of the coupling constant in the perturbative expansion of the observables. For a long time, physicists have been aware that perturbative expansions have a limited scope, in spite of all the remarkable achievements obtained with them. Some examples of such situations are resonant effects associated to the coupling of atoms to strong radiofrequency fields, or the low-energy domain of quantum chromodynamics, where confinement of quarks and gluons takes place. Along the last decades, many attempts were devised to circumvent the limitations of perturbation theory, in particular when strong effective couplings are involved.

In any case, as a matter of principle, due to the nonvanishing of the coupling constant, the idea of a bare particle associated to a bare matter field is actually an artifact of perturbation theory and strictly speaking is physically meaningless. In general, a charged physical particle is always coupled to a gauge field; in other words, it is always "dressed" by a cloud of quanta of the gauge field, for instance, photons in the case of electrodynamics.

With respect to the time evolution of systems of (matter) particles, the idea is that particles are coupled to an environment or to a thermal bath. There are usually two equivalent ways of modeling the environment to which the particle is coupled: either to represent it by a free field, as was done in Refs. [1, 2], or to consider the environment as a reservoir composed of a large number of noninteracting harmonic oscillators (see, for instance, [3, 4, 5, 6]). In both cases, exactly the same type of argument given above for a charged particle applies, making the appropriate changes, to these systems. We may speak of a "dressing" of the set of particles by the ensemble of the harmonic modes of the environment. The particles in the system are considered as always "dressed" by a cloud of quanta of the environment. This is true in general for any system in which material particles are coupled to an environment, no matter what the specific nature of the environment and interaction involved are.

In the present work we adopt this point of view and consider a harmonic oscillator, coupled linearly to an environment modeled by the infinite set of harmonic modes of a scalar field, the whole system being contained in a perfectly reflecting sphere of radius $R$. We implement a nonperturbative study of the time evolution of a superposition of the first excited and the ground states of the oscillator, by means of the dressed states introduced in [7] and already employed to investigate several situations [8, 9, 10, 11, 12, 13. Such a nonperturbative approach is possible due to the linear character of the interaction between the oscillator and the environment we choose.

The semiqualitative idea of a "dressed atom" was originally introduced in Refs. [14] and [15], and largely employed in studies involving the interaction of atoms and electromagnetic fields [18, 19, 20, 21]. In the realm of general physics, the concept 
of dressing a matter particle by an environment has found an application in describing the radiation damping of classical systems [16]. Our dressed states can be viewed as a rigorous version of these dressing procedures. Moreover, the oscillator may represent a mode of the quantized electromagnetic field in a cavity interacting with the environment; in this case, our study refers to the time evolution of the superposition of the ground state and the first excited state of the field in the cavity.

The model in itself is not new; a system composed of a material body interacting linearly with an environment has been the main subject of many papers, as those quoted in Refs. [1, 2, 3, 4, 15, 6, 17, 16]. The novelty lies in the nonperturbative approach to the problem, by means of renormalized coordinates and dressed states, which was started in [7]. In particular, our dressed states are not the same as those employed in the literature, usually associated to normal coordinates. Our dressed states are given in terms of our renormalized coordinates and allow a rigorous study of the time evolution of quantum systems. The results we obtain by this means are those expected on physical grounds, but contain corrections with respect to the formulas obtained from perturbation theory.

We structure the paper as follows. In Section II we review the basic aspects of the formalism of renormalized coordinates and dressed states and then, in Section III, we treat the time evolution of a superposition of oscillator states in both limiting situations of $R \rightarrow \infty$ and small $R$. Section IV contains our final comments.

\section{Renormalized coordinates and dressed states}

Let us start by considering the harmonic oscillator having bare frequency $\omega_{0}$, linearly coupled to a field described by $N(\rightarrow \infty)$ other oscillators, with frequencies $\omega_{k}$, $k=1,2, \ldots, N$. The whole system is contained in a perfectly reflecting spherical cavity of radius $R$, the free space corresponding to the limit $R \rightarrow \infty$. Hereafter, we shall refer to the harmonic oscillator as the particle, to distinguish it from the harmonic modes of the environment. Denoting by $q_{0}(t)\left(p_{0}(t)\right)$ and $q_{k}(t)\left(p_{k}(t)\right)$ the coordinates (momenta) associated with the particle and the field oscillators respectively, the Hamiltonian of the system is

$$
H=\frac{1}{2}\left[p_{0}^{2}+\omega_{0}^{2} q_{0}^{2}+\sum_{k=1}^{N}\left(p_{k}^{2}+\omega_{k}^{2} q_{k}^{2}\right)\right]-q_{0} \sum_{k=1}^{N} \eta \omega_{k} q_{k},
$$

where the limit $N \rightarrow \infty$ is understood and $\eta$ is a constant.

The Hamiltonian (1) can be turned to principal axis by means of a point transformation,

$$
q_{\mu}=\sum_{r=0}^{N} t_{\mu}^{r} Q_{r}, \quad p_{\mu}=\sum_{r=0}^{N} t_{\mu}^{r} P_{r}
$$

where $\mu=(0,\{k\}), k=1,2, \ldots, N$, performed by an orthonormal matrix $T=\left(t_{\mu}^{r}\right)$. The subscripts $\mu=0$ and $\mu=k$ refer respectively to the particle and the harmonic 
modes of the field and $r$ refers to the normal modes. In terms of normal momenta and coordinates, the transformed Hamiltonian reads

$$
H=\frac{1}{2} \sum_{r=0}^{N}\left(P_{r}^{2}+\Omega_{r}^{2} Q_{r}^{2}\right)
$$

where the $\Omega_{r}$ 's are the normal frequencies corresponding to the collective stable oscillation modes of the coupled system.

Using the coordinate transformation (2) in the equations of motion and explicitly making use of the normalization condition

$$
\sum_{\mu=0}^{N}\left(t_{\mu}^{r}\right)^{2}=1
$$

we get

$$
t_{k}^{r}=\frac{\eta \omega_{k}}{\omega_{k}^{2}-\Omega_{r}^{2}} t_{0}^{r}, \quad t_{0}^{r}=\left[1+\sum_{k=1}^{N} \frac{\eta^{2} \omega_{k}^{2}}{\left(\omega_{k}^{2}-\Omega_{r}^{2}\right)^{2}}\right]^{-\frac{1}{2}}
$$

with the condition

$$
\omega_{0}^{2}-\Omega_{r}^{2}=\sum_{k=1}^{N} \frac{\eta^{2} \omega_{k}^{2}}{\omega_{k}^{2}-\Omega_{r}^{2}}
$$

The right hand side of Eq. (6) diverges in the limit $N \rightarrow \infty$. Defining the counterterm $\delta \omega^{2}=N \eta^{2}$, it can be rewritten in the form

$$
\omega_{0}^{2}-\delta \omega^{2}-\Omega_{r}^{2}=\eta^{2} \Omega_{r}^{2} \sum_{k=1}^{N} \frac{1}{\omega_{k}^{2}-\Omega_{r}^{2}} .
$$

Equation (77) has $N+1$ solutions, corresponding to the $N+1$ normal collective modes. It can be shown [7] that if $\omega_{0}^{2}>\delta \omega^{2}$, all possible solutions for $\Omega^{2}$ are positive, physically meaning that the system oscillates harmonically in all its modes. On the other hand, when $\omega_{0}^{2}<\delta \omega^{2}$, one of the solutions is negative and so no stationary configuration is allowed. Nevertheless, in a different context, it is precisely this runaway solution that is related to the existence of a bound state in the Lee-Friedrichs model. This solution is considered in Ref. [22] in the framework of a model to describe qualitatively the existence of bound states in particle physics.

Therefore, we just consider the situation in which all normal modes are harmonic, which corresponds to the first case above, $\omega_{0}^{2}>\delta \omega^{2}$, and define the renormalized frequency

$$
\bar{\omega}^{2}=\lim _{N \rightarrow \infty}\left(\omega_{0}^{2}-N \eta^{2}\right),
$$

following the pioneering work of Ref. [23]. In the limit $N \rightarrow \infty$, Eq. (7) becomes

$$
\bar{\omega}^{2}-\Omega^{2}=\eta^{2} \sum_{k=1}^{\infty} \frac{\Omega^{2}}{\omega_{k}^{2}-\Omega^{2}} .
$$

We see that, in this limit, the above procedure is exactly the analogue of mass renormalization in quantum field theory: the addition of a counterterm $-N \eta^{2} q_{0}^{2}$ allows 
one to compensate the infinity of $\omega_{0}^{2}$ in such a way as to leave a finite, physically meaningful, renormalized frequency $\bar{\omega}$.

To proceed, we take the constant $\eta$ as

$$
\eta=\sqrt{\frac{4 g \Delta \omega}{\pi}}
$$

where $\Delta \omega$ is the interval between two neighboring field frequencies and $g$ is the coupling constant with dimension of frequency. The environment frequencies $\omega_{k}$ can be written in the form

$$
\omega_{k}=k \frac{\pi c}{R}, \quad k=1,2, \ldots,
$$

and, so, $\Delta \omega=\pi c / R$. Then, using the identity

$$
\sum_{k=1}^{\infty} \frac{1}{k^{2}-u^{2}}=\frac{1}{2}\left[\frac{1}{u^{2}}-\frac{\pi}{u} \cot (\pi u)\right]
$$

Eq. (9) can be written in closed form:

$$
\cot \left(\frac{R \Omega}{c}\right)=\frac{\Omega}{2 g}+\frac{c}{R \Omega}\left(1-\frac{R \bar{\omega}^{2}}{2 g c}\right) .
$$

The elements of the transformation matrix, turning the particle-field system to principal axis, are obtained in terms of the physically meaningful quantities $\Omega_{r}, \bar{\omega}$, after some rather long but straightforward manipulations [7]. They read

$$
\begin{aligned}
t_{0}^{r} & =\frac{\eta \Omega_{r}}{\sqrt{\left(\Omega_{r}^{2}-\bar{\omega}^{2}\right)^{2}+\frac{\eta^{2}}{2}\left(3 \Omega_{r}^{2}-\bar{\omega}^{2}\right)+4 g^{2} \Omega_{r}^{2}}}, \\
t_{k}^{r} & =\frac{\eta \omega_{k}}{\omega_{k}^{2}-\Omega_{r}^{2}} t_{0}^{r} .
\end{aligned}
$$

Let us now consider the eigenstates of our system, $\left|n_{0}, n_{1}, n_{2}, \ldots\right\rangle$, represented by the normalized eigenfunctions, written in terms of the normal coordinates $\left\{Q_{r}\right\}$,

$$
\phi_{l_{0} l_{1} l_{2} \ldots}(Q, t)=\prod_{s}\left[\sqrt{\frac{2^{l_{s}}}{l_{s} !}} H_{l_{s}}\left(\sqrt{\frac{\Omega_{s}}{\hbar}} Q_{s}\right)\right] \Gamma_{0} e^{-i \sum_{s}\left(l_{s}+\frac{1}{2}\right) \Omega_{s} t},
$$

where $H_{l_{s}}$ stands for the $l_{s}$-th Hermite polynomial and $\Gamma_{0}$ is the normalized vacuum eigenfunction.

We introduce renormalized coordinates $q_{0}^{\prime}$ and $\left\{q_{i}^{\prime}\right\}$ for the dressed particle and the dressed field, respectively, defined by

$$
\sqrt{\bar{\omega}_{\mu}} q_{\mu}^{\prime}=\sum_{r} t_{\mu}^{r} \sqrt{\Omega_{r}} Q_{r}
$$

where $\bar{\omega}_{\mu}=\left\{\bar{\omega}, \omega_{i}\right\}$. In terms of the renormalized coordinates, we define for a fixed instant, $t=0$, dressed states, $\left|\kappa_{0}, \kappa_{1}, \kappa_{2}, \ldots\right\rangle$ by means of the complete orthonormal set of functions [7]

$$
\psi_{\kappa_{0} \kappa_{1} \ldots}\left(q^{\prime}\right)=\prod_{\mu}\left[\sqrt{\frac{2^{\kappa_{\mu}}}{\kappa_{\mu} !}} H_{\kappa_{\mu}}\left(\sqrt{\frac{\bar{\omega}_{\mu}}{\hbar}} q_{\mu}^{\prime}\right)\right] \Gamma_{0},
$$


where $q_{\mu}^{\prime}=\left\{q_{0}^{\prime}, q_{i}^{\prime}\right\}$. Note that the ground state $\Gamma_{0}$ in the above equation is the same as in Eq. (16). The invariance of the ground state is due to our definition of renormalized coordinates given by Eq. (17). Each function $\psi_{\kappa_{0} \kappa_{1} \ldots}\left(q^{\prime}\right)$ describes a state in which the dressed oscillator $q_{\mu}^{\prime}$ is in its $\kappa_{\mu}$-th excited state. In terms of the bare coordinates, the renormalized coordinates are expressed as

$$
q_{\mu}^{\prime}=\sum_{\nu} \alpha_{\mu \nu} q_{\nu}
$$

where

$$
\alpha_{\mu \nu}=\frac{1}{\sqrt{\bar{\omega}_{\mu}}} \sum_{r} t_{\mu}^{r} t_{\nu}^{r} \sqrt{\Omega_{r}} .
$$

Remark that the introduction of the renormalized coordinates implies, differently from the bare vacuum, the stability of the dressed vacuum state since, by construction, it is identical to the ground state of the interacting Hamiltonian (3). Also it is important to notice that the renormalized coordinates and the dressed states, defined in Eqs. (17) and (18) are new collective objects, different from the normal coordinates $Q$ and the eigenstates (16). Since the transformation (17) is not orthogonal, the Hamiltonian is not diagonal in the renormalized coordinates. Thus, distinctly from the eigenstates, our dressed states are all unstable, except for the ground dressed $\left(\left\{\kappa_{\mu}=0\right\}\right)$ state. We shall assume that the dressed states (18) are the physically appropriate states to describe the time evolution of superpositions of states of the mechanical oscillator, taking into account non-perturbatively the effect of the interaction with the environment. This is an alternative to the use of states written in terms of the bare coordinates $q_{\mu}$, which would require a perturbative renormalization procedure to correct order by order the oscillator frequency.

Let us consider, for instance, the particular dressed state $\left|\Gamma_{1}^{\mu}(0)\right\rangle$, represented by the wave function $\psi_{00 \cdots 1(\mu) 0 \cdots}\left(q^{\prime}\right)$. It can be seen as describing, at a given instant, the configuration in which only the $\mu$-th dressed oscillator is in the "first excited level", all others being in their "ground states". These "levels" should not be confused with the stationary states given by Eq. (16); from now on, we shall use such a terminology to facilitate the reference to dressed states. As shown in Ref. [7, the time evolution of the state $\left|\Gamma_{1}^{\mu}\right\rangle$ is given by

$$
\left|\Gamma_{1}^{\mu}(t)\right\rangle=\sum_{\nu} f_{\mu \nu}(t)\left|\Gamma_{1}^{\nu}(0)\right\rangle,
$$

where

$$
f_{\mu \nu}(t)=\sum_{s} t_{\mu}^{s} t_{\nu}^{s} e^{-i \Omega_{s} t} .
$$

Moreover, it can be shown that, for all $\mu$,

$$
\sum_{\nu}\left|f_{\mu \nu}(t)\right|^{2}=1,
$$

which allows to interpret the coefficients $f_{\mu \nu}(t)$ as probability amplitudes; for example, $f_{00}(t)$ is the probability amplitude that, if the dressed particle is in the first excited state 
at $t=0$, it remains excited at time $t$, while $f_{0 i}(t)$ represents the probability amplitude that the $i$-th dressed harmonic mode of the cavity be at the first excited level. Also, the elements of the matrix $T_{\kappa_{0} \kappa_{1} \ldots}^{l_{0} l_{1} \ldots}$, connecting the dressed states to the eigenstates, can be evaluated. For the dressed state in which only the $\kappa_{\mu}$-th dressed oscillator is in the $N$-th excited level, all other being in the ground state, we have [7],

$$
T_{0,0, \cdots}^{l_{0} l_{1} \cdots}=\left(\frac{N !}{l_{0} ! l_{1} ! \cdots}\right)^{\frac{1}{2}}\left(t_{\mu}^{0}\right)^{l_{0}}\left(t_{\mu}^{1}\right)^{l_{1}} \cdots
$$

where $l_{0}+l_{1}+\cdots=N$.

\section{Behavior of the superposition of states in a cavity}

We now analyze the situation in which, at time $t=0$, the environment oscillators are in their ground states and the particle is in a superposition of dressed states, and the whole system is allowed to evolve in time. Specifically, we take, as the initial particle dressed state, an arbitrary superposition of the first-excited and the ground states,

$$
|\psi\rangle=\sqrt{\xi}\left|\Gamma_{1}^{0}(0)\right\rangle+\sqrt{1-\xi} e^{i \phi}\left|\Gamma_{0}\right\rangle
$$

where $0<\xi<1$. While the ground state is stable, the particle first-excited state evolves in time according to Eq. (21), that is, $\left|\Gamma_{1}^{0}(t)\right\rangle=\sum_{\nu} f_{0 \nu}(t)\left|\Gamma_{1}^{\nu}(0)\right\rangle$ with

$f_{0 \nu}(t)=\sum_{s} t_{0}^{s} t_{\nu}^{s} e^{-i \Omega_{s} t}$. At the instant $t$, the state of the system is given by the density matrix

$$
\varrho(t)=e^{-i H t}|\psi\rangle\langle\psi| e^{i H t}
$$

We are interested in studying the influence of the environment on the time evolution of the state of the subsystem corresponding to the particle. Thus, we take the trace over all the degrees of freedom associated with the field, that is, we consider the reduced density matrix

$$
\rho(t)=\sum_{\left\{k_{i}=0\right\}}^{\infty}\left\langle k_{1}, k_{2}, \ldots\left|e^{-i H t}\right| \psi\right\rangle\left\langle\psi\left|e^{i H t}\right| k_{1}, k_{2}, \ldots\right\rangle,
$$

whose elements between dressed particle states are

$$
\rho_{m n}(t)=\langle m|\rho| n\rangle=\sum_{\left\{k_{i}=0\right\}}^{\infty}\left\langle m, k_{1}, k_{2}, \ldots\left|e^{-i H t}\right| \psi\right\rangle\left\langle\psi\left|e^{i H t}\right| n, k_{1}, k_{2}, \ldots\right\rangle .
$$

Replacing Eq. (25) in Eq. (28) we get

$$
\begin{aligned}
\rho_{m n}(t)= & \sum_{\left\{k_{i}=0\right\}}^{\infty}\left\{\xi \mathcal{A}_{100 \ldots}^{m k_{1} k_{2} \ldots}(t) \mathcal{A}_{100 \ldots}^{n k_{1} k_{2} \ldots *}(t)+(1-\xi) \mathcal{A}_{000 \ldots}^{m k_{1} k_{2} \ldots}(t) \mathcal{A}_{000 \ldots}^{n k_{1} k_{2} \ldots *}(t)\right. \\
& +\sqrt{\xi(1-\xi)} e^{-i \phi} \mathcal{A}_{100 \ldots}^{m k_{1} k_{2} \ldots}(t) \mathcal{A}_{000 \ldots}^{n k_{1} k_{2} \ldots *}(t) \\
& \left.+\sqrt{\xi(1-\xi)} e^{i \phi} \mathcal{A}_{000 \ldots}^{m k_{1} k_{2} \ldots}(t) \mathcal{A}_{100 \ldots}^{n k_{1} k_{2} \ldots *}(t)\right\}
\end{aligned}
$$

where

$$
\mathcal{A}_{n_{0} n_{1} n_{2} \ldots}^{m_{0} m_{1} m_{2} \ldots}(t)=\left\langle m_{0}, m_{1}, m_{2}, \ldots\left|e^{-i H t}\right| n_{0}, n_{1}, n_{2}, \ldots\right\rangle
$$


are the probability amplitudes between the dressed states. These probability amplitudes can be computed using Eqs. (22) and (24). We then have

$$
\begin{aligned}
& \mathcal{A}_{000 \ldots}^{m_{0} m_{1} m_{2} \cdots}(t)=e^{-i E_{0} t} \delta_{0 m_{0}} \delta_{0 m_{1}} \delta_{0 m_{2}} \ldots \\
& \mathcal{A}_{100 \ldots}^{m_{0} m_{1} m_{2} \ldots}(t)=e^{-i E_{0} t} \sum_{\mu} f_{0 \mu}(t) \delta_{1 m_{\mu}} \prod_{\nu \neq \mu} \delta_{0 m_{\nu}},
\end{aligned}
$$

where $E_{0}=\sum_{r} \Omega_{r} / 2$ is the ground state energy of the system. Substituting the above equations into Eq. (29), we obtain, after summing over the $k_{i}$ 's,

$$
\begin{aligned}
\rho_{m n}(t)= & \left(1-\xi+\xi \sum_{k}\left|f_{0 k}(t)\right|^{2}\right) \delta_{0 m} \delta_{0 n}+\xi\left|f_{00}(t)\right|^{2} \delta_{1 m} \delta_{1 n} \\
& +\sqrt{\xi(1-\xi)} e^{-i \phi} f_{00}(t) \delta_{1 m} \delta_{0 n}+\sqrt{\xi(1-\xi)} e^{i \phi} f_{00}^{*}(t) \delta_{0 m} \delta_{1 n}
\end{aligned}
$$

Using that $\sum_{\mu}\left|f_{0 \mu}\right|^{2}=1$, the nonzero elements of the reduced density matrix are given by

$$
\begin{aligned}
& \rho_{00}(t)=1-\xi\left|f_{00}(t)\right|^{2}, \\
& \rho_{11}(t)=\xi\left|f_{00}(t)\right|^{2}, \\
& \rho_{10}(t)=\sqrt{\xi(1-\xi)} e^{-i \phi} f_{00}(t), \\
& \rho_{01}(t)=\sqrt{\xi(1-\xi)} e^{i \phi} f_{00}^{*}(t) .
\end{aligned}
$$

Note that $\operatorname{Tr}[\rho(t)]=1$, which means that the reduced density matrix $\rho(t)$ does represent a physical state of the particle; also, it is not a pure state, since $\operatorname{Tr}\left[\rho^{2}(t)\right] \neq 1$. The degree of impurity of a quantum state can be quantified by the departure of the idempotent property; in the present case, we have

$$
D(t ; \xi)=1-\operatorname{Tr}\left[\rho^{2}\right]=2 \xi^{2}\left|f_{00}(t)\right|^{2}\left(1-\left|f_{00}(t)\right|^{2}\right) .
$$

We are thus left with the calculation of the quantity $f_{00}(t)$, which will be done for the particular situations of an arbitrarily large cavity $(R \rightarrow \infty)$ and of a small cavity.

\subsection{Large cavity}

For an arbitrarily large value of $R$, Eq. (14) reduces to

$$
t_{0}^{r} \rightarrow \lim _{R \rightarrow \infty} \frac{\sqrt{4 g / \pi} \Omega \sqrt{\pi c / R}}{\sqrt{\left(\Omega^{2}-\bar{\omega}^{2}\right)^{2}+4 g^{2} \Omega^{2}}} .
$$

Also, in this limit, $\Delta \omega=\pi c / R \rightarrow d \omega=d \Omega$ and the expression for the quantity $f_{00}(t)$, given by Eq. (22), can be cast in the form

$$
f_{00}(t)=\frac{4 g}{\pi} \int_{0}^{\infty} d \Omega \frac{\Omega^{2} e^{-i \Omega t}}{\left(\Omega^{2}-\bar{\omega}^{2}\right)^{2}+4 g^{2} \Omega^{2}} .
$$

The real part of $f_{00}(t)$ can be evaluated directly using the residue theorem. Defining the parameter $\kappa$ by

$$
\kappa=\sqrt{\bar{\omega}^{2}-g^{2}},
$$


we obtain, for the three distinct cases,

a) $\kappa^{2}>0$ :

$$
f_{00}(t)=e^{-g t}\left[\cos \kappa t-\frac{g}{\kappa} \sin \kappa t\right]+i G(t ; \bar{\omega}, g)
$$

b) $\kappa^{2}=0$ :

$$
f_{00}(t)=e^{-g t}[1-g t]+i G(t ; \bar{\omega}, g) ;
$$

and c) $\kappa^{2}<0$ :

$$
f_{00}(t)=e^{-g t}\left[\cosh |\kappa| t-\frac{g}{|\kappa|} \sinh |\kappa| t\right]+i G(t ; \bar{\omega}, g),
$$

where the function $G(t ; \bar{\omega}, g)$ is given by

$$
G(t ; \bar{\omega}, g)=-\frac{4 g}{\pi} \int_{0}^{\infty} d y \frac{y^{2} \sin y t}{\left(y^{2}-\bar{\omega}^{2}\right)^{2}+4 g^{2} y^{2}} .
$$

The overall behavior of the function $G(t ; \bar{\omega}, g)$ is illustrated in Fig. 1; we see then that, in all cases, the real and imaginary parts of $f_{00}(t)$ decay with the time, for large times. This aspect dictates the behavior of the degree of purity, as a function of time, as illustrated in Fig. 2, for a case with $g<\bar{\omega}$; similar results are obtained for the other regimes.

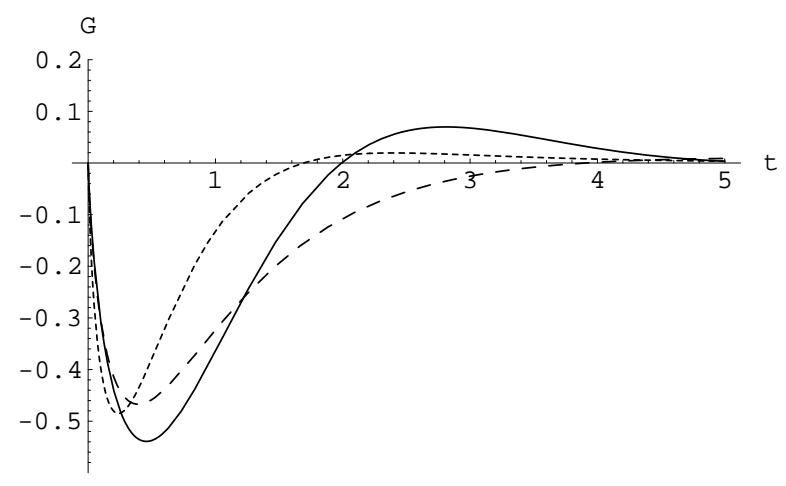

Figure 1. Behavior of $G(t ; \bar{\omega}, g)$ as a function of $t$ for the three distinct cases: $\bar{\omega}=1.5$ and $g=1.0$ (full line); $\bar{\omega}=1.0$ and $g=1.2$ (dashed line); and $\bar{\omega}=g=2.0$ (dotted line), in arbitrary units.

Replacing $f_{00}(t)$, given by (43), (44) or (45), into Eqs. (35)-(38) leads to the elements of the reduced density matrix; simpler expressions are obtained for large times $(t \gg 1 / \bar{\omega})$ at both weak- and strong-coupling regimes. For large $t, G(t ; \bar{\omega}, g)$ can be approximated as

$$
G(t ; \bar{\omega}, g) \approx \frac{8 g}{\pi \bar{\omega}^{4} t^{3}} \quad\left(t \gg \frac{1}{\bar{\omega}}\right) .
$$

Thus, in the limit case of weak coupling between the particle and the environment, $g \ll \bar{\omega}$ (corresponding to $\kappa \approx \bar{\omega}$ ), the large-time approximation of the reduced density 


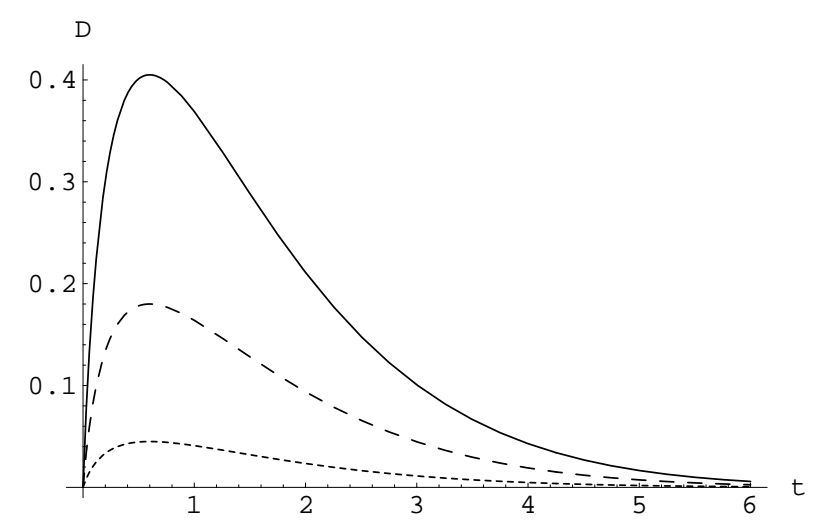

Figure 2. Behavior of $D(t ; \xi)$ as a function of $t$, with $\bar{\omega}=1.0$ and $g=0.5$ fixed (in arbitrary units), for some values of $\xi: 0.3,0.6$ and 0.9 (dotted, dashed and full lines, respectively).

matrix gives

$$
\begin{aligned}
& \rho_{11}(t) \approx \xi\left\{e^{-2 g t}\left[\cos \bar{\omega} t-\frac{g}{\bar{\omega}} \sin \bar{\omega} t\right]^{2}+\frac{64 g^{2}}{\pi^{2} \bar{\omega}^{8} t^{6}}\right\}, \\
& \rho_{00}(t)=1-\rho_{11}(t), \\
& \rho_{10}(t) \approx \sqrt{\xi(1-\xi)} e^{-i \phi}\left\{e^{-g t}\left[\cos \bar{\omega} t-\frac{g}{\bar{\omega}} \sin \bar{\omega} t\right]+i \frac{8 g}{\pi \bar{\omega}^{4} t^{3}}\right\}, \\
& \rho_{01}(t)=\rho_{10}^{*}(t) .
\end{aligned}
$$

Similar results can be obtained for the case of a strong coupling between the particle and the environment, that is, when $g \gg \bar{\omega}$ (i.e. $|\kappa| \approx g$ ); for large $t$, we find

$$
\begin{aligned}
& \rho_{11}(t) \approx \xi\left[e^{-4 g t}+\frac{64 g^{2}}{\pi^{2} \bar{\omega}^{8} t^{6}}\right], \\
& \rho_{00}(t)=1-\rho_{11}(t), \\
& \rho_{10}(t) \approx \sqrt{\xi(1-\xi)} e^{-i \phi}\left[e^{-2 g t}+i \frac{8 g}{\pi \bar{\omega}^{4} t^{3}}\right], \\
& \rho_{01}(t)=\rho_{10}^{*}(t) .
\end{aligned}
$$

We see from the above equations that, for both weak- and strong-coupling regimes, in the case of a very large cavity, except for the vacuum-vacuum component which goes to 1 , all other matrix elements of the reduced density matrix tend to zero as $t \rightarrow \infty$. This means that the particle mixed state $\rho(t)$ tends to the dressed-particle vacuum state as time evolves; thus, for large cavities, the particle system is dissipative. This is also illustrated in Fig. 2, with $D(t ; \xi)$ vanishing as $t \rightarrow \infty$ for all values of $\xi$. Notice that the the real and imaginary parts of the matrix elements $\rho_{10}(t)$ and $\rho_{01}(t)$, which corresponds to interference terms, decay more slowly then the element $\rho_{11}(t)$. 


\subsection{Small cavity}

For a finite cavity, the spectrum of eigenfrequencies is discrete, $\Delta \omega$ is large, and so the approximation made in the case of large cavity does not apply; no analytical result can be obtained for $f_{00}(t)$, given by Eq. (22), in this case. However, if the cavity is sufficiently small, the frequencies $\Omega_{r}$ can be determined as follows [8]. In terms of the small dimensionless parameter

$$
\delta=\frac{g}{\Delta \omega}=\frac{g R}{\pi c}
$$

Eq. (13) is rewritten as

$$
\cot (\pi \theta)=\frac{\theta}{2 \delta}+\frac{1}{\pi \theta}\left(1-\frac{\pi \bar{\omega}^{2} \delta}{2 g^{2}}\right)
$$

where $\theta=\Omega / \Delta \omega$. With $\delta \ll 1$, which corresponds to $R \ll \pi c / g$ (a small cavity), as plots of both sides of Eq. (157) as functions of $\theta$ are drawn, one sees that, for $k=1,2, \ldots$, the solutions of Eq. (57) can be written as $\theta_{k} \approx k+\epsilon_{k}$ with $\epsilon_{k} \ll 1$; then, expanding $\cot \left(\pi \epsilon_{k}\right)$ for small $\epsilon_{k}$, one finds

$$
\Omega_{k} \approx \Delta \omega\left(k+\frac{2 \delta}{\pi k}\right)=\frac{g}{\delta}\left(k+\frac{2 \delta}{\pi k}\right) .
$$

If we further impose that $\delta<2 g^{2} / \pi \bar{\omega}^{2}$, a condition compatible with $\delta \ll 1$, then $\Omega_{0}$ is found to be very close to $\bar{\omega}$, that is,

$$
\Omega_{0} \approx \bar{\omega}\left(1-\frac{\pi \delta}{2}\right)
$$

To determine $f_{00}(t)$, we have to calculate the square of the matrix elements $\left(t_{0}^{0}\right)^{2}$ and $\left(t_{k}^{0}\right)^{2}$. From Eq. (15), using that $\omega_{k}=k \Delta \omega=k g / \delta$, we find (to first order in $\delta$ )

$$
\left(t_{k}^{0}\right)^{2} \approx \frac{4 \delta}{\pi k^{2}}\left(t_{0}^{0}\right)^{2}
$$

Now, considering the orthonormalization condition (41), we determine $\left(t_{0}^{0}\right)^{2}$ as

$$
\left(t_{0}^{0}\right)^{2} \approx\left(1+\frac{4 \delta}{\pi} \sum_{k=1}^{\infty} k^{-2}\right)^{-1}=\left(1+\frac{2}{3} \pi \delta\right)^{-1}
$$

where we have used that $\zeta(2)=\sum_{k=1}^{\infty} k^{-2}=\pi^{2} / 6$. We thus obtain, for sufficiently small cavities $(\delta \ll 1)$,

$$
f_{00}(t) \approx\left(1+\frac{2}{3} \pi \delta\right)^{-1}\left[e^{-i \bar{\omega}\left(1-\frac{\pi \delta}{2}\right) t}+\sum_{k=1}^{\infty} \frac{4 \delta}{\pi k^{2}} e^{-i \frac{g}{\delta}\left(k+\frac{2 \delta}{\pi k}\right) t}\right] .
$$

Notice that, if we had calculated $\left(t_{0}^{0}\right)^{2}$ by approximating directly Eq. (14) for small $\delta$, instead of using the normalization condition (4), $f_{00}(t)$ would not satisfy the requirement $\left|f_{00}(t)\right|^{2} \leq 1$ with the equality occurring when $t=0$. Also, it is worth mentioning that the approximation we made holds independently of the strength of the coupling between the particle and the environment and, thus, it applies for both weak and strong limits. 
Substituting $f_{00}(t)$, given by Eq. (62), into Eqs. (35)-(38) leads to the elements of the reduced density matrix for the case of a small cavity. Distinctly from the case of a very large cavity, now, these matrix elements do not tend to zero as time evolves; in fact, they oscillate, never reaching zero. In particular, we find that

$$
\begin{aligned}
\rho_{11}(t) \approx & \left(1+\frac{2}{3} \pi \delta\right)^{-2}\left\{1+\frac{8 \delta}{\pi} \sum_{k=1}^{\infty} \frac{1}{k^{2}} \cos \left[\bar{\omega}\left(1-\frac{\pi \delta}{2}\right)-\frac{g}{\delta}\left(k+\frac{2 \delta}{\pi k}\right)\right] t\right. \\
& \left.+\frac{16 \delta^{2}}{\pi^{2}} \sum_{k, l=1}^{\infty} \frac{1}{k^{2} l^{2}} \cos \left[\left(\frac{g}{\delta}-\frac{2 g}{\pi k l}\right)(k-l)\right] t\right\} .
\end{aligned}
$$

Note that $\rho_{11}(t)$ is an oscillating, nonperiodic, function of time. A lower bound for $\rho_{11}$ can be found if one replaces the cosine terms in Eq. (63) by -1 ; in fact, to order $\delta^{2}$, one finds

$$
\rho_{11}(t)>\xi\left[1-\frac{8}{3} \pi \delta+\frac{8}{9} \pi^{2} \delta^{2}\right] .
$$

This means that the particle state never reduces to the dressed-particle vacuum state; the particle system, maintaining itself in a mixed state, keeps exchanging quanta with the environment.

The degree of impurity of this particle state at time $t$ can be calculated using Eq. (39) or, equivalently, with

$$
D(t ; \xi)=2 \rho_{11}(t)\left[\xi-\rho_{11}(t)\right] .
$$

This quantity is plotted in Fig. 3, for the same values of $\bar{\omega}, g$ and $\xi$ as those used in Fig. 2 (for comparison), fixing a specific value of $\delta$; one clearly sees the oscillatory, nonperiodic, behavior mentioned above. Therefore, in the case of a small cavity, for both weak- and strong-coupling regimes, the particle system is not dissipative; it keeps exchanging energy with the environment. For example, in the case of weak coupling, a physically interesting situation occurs when interactions of electromagnetic type are involved. In this case, we can take $g=\alpha \bar{\omega}$, where $\alpha$ is the fine-structure constant, $\alpha \sim 1 / 137$. For a frequency $\bar{\omega} \sim 2 \times 10^{11} / \mathrm{s}$ (in the microwave band) and a cavity with $R \sim 10^{-2} \mathrm{~m}$, we have $\delta \sim 0.016$ and, so, the diagonal element $\rho_{11}(t)$ will never fall below the value $\sim 0.87 \xi$. For strong coupling, $g=\beta \bar{\omega}(\beta>1)$, a similar result holds for a

smaller cavity. The same kind of oscillatory behavior can be verified for the nondiagonal elements of the density matrix. In other words, the system confined in a small cavity is not dissipative, contrary to the case of a large cavity.

\section{Concluding Remarks}

We have presented in this paper a nonperturbative treatment of a quantum system consisting of a particle, in the harmonic approximation, coupled to an environment modeled by noninteracting oscillators. We have used renormalized coordinates in terms of which dressed states can be constructed. These states allow to naturally separate the system into the dressed (physically observed) particle and the dressed 


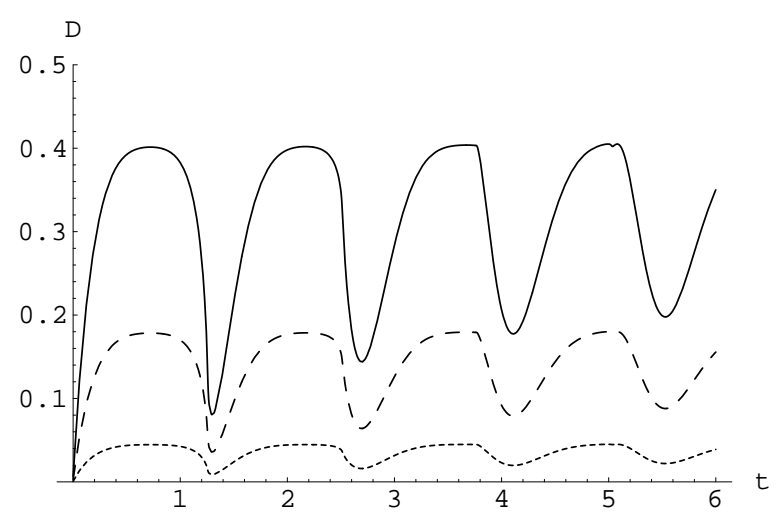

Figure 3. Behavior of $D(t ; \xi)$ as a function of $t$ for $\delta=0.1$, with $\bar{\omega}=1.0$ and $g=0.5$ fixed (in arbitrary units), for some values of $\xi: 0.3,0.6$ and 0.9 (dotted, dashed and full lines, respectively).

environment by means of the conveniently chosen renormalized coordinates, $q_{0}^{\prime}$ and $q_{j}^{\prime}$, associated respectively to the dressed particle and to the dressed oscillators composing the environment. The dressed particle will contain automatically all the effects of the environment in it. Using this formalism we perform a nonperturbative study of the time evolution of a superposition of the ground state and the first excited state of the particle, the system being confined in a perfectly reflecting cavity of radius $R$.

In the $R \rightarrow \infty$ limit, we find dissipation (damping) with dominance of the interference terms of the density matrix, in both weak- and strong-coupling regimes. For small values of $R$, the system is not dissipative, presenting stability. In both cases we have obtained results in agreement with expected behaviours. However, we do not simply recover well-known features with our method. For instance, as far as we know, the corrections to the exponential decay in Eqs. (48) to (51) and from Eqs. (52) to (55) have not been reported before in the literature. Also, the absence of dissipation in a small cavity can be understood as the analogue of spontaneous emission inhibition for an atom, which has a qualitative explanation when the lowest resonance cavity frequency is larger than the oscillator frequency. But also in this case we are able, with our method, to give rigorous expressions for the elements of the density matrix (in an analogous manner as has been done in 8 ] for the size of cavities ensuring stability of excited atoms).

It is worth to stress that the renormalized coordinates and the dressed states, defined in Eqs. (17) and (18), allow an exact treatment of the problem, in which one completely avoids the use of perturbation theory. As already noticed in the text below Eq. (20), the renormalized coordinates and dressed states are new collective objects, different from the normal coordinates $Q$ and the eigenstates (16). Distinctly from the eigenstates, our dressed states are all unstable, except for the ground dressed $\left(\left\{\kappa_{\mu}=0\right\}\right)$ state. We assume that the dressed states (18) are the physically meaningful states, instead of the ones written in terms of the bare coordinates $q_{\mu}$. This can be seen as 
analogous to the wave-function renormalization in quantum field theory, which justifies referring to $q_{\mu}^{\prime}$ as renormalized coordinates. Also, it is worth mentioning that the introduction of renormalized coordinates naturally ensures that the dressed vacuum state is stable, contrarily to the bare perturbative vacuum, since, by construction, it is identical to the ground state of the interacting Hamiltonian (3). Remember that the invariance of the ground state is due to our definition of renormalized coordinates given by Eq. (17).

As already explained in the Introduction, our formalism and results are restricted to a linear model, but it has the advantage of being nonperturbatively solvable. More realistic physical situations, however, would require a nonlinear coupling between the particle and the environment. It is nevertheless envisageable that such a study of complex nonlinear systems could be handled with our nonperturbative method. Indeed, the concept of renormalized coordinates has already been extended to a nonlinear model in Ref. [12. Also, the generalization of the work presented in the present paper to the case of finite temperature, as well as the study of dressed coherent states, is in progress and will be presented elsewhere.

\section{Acknowledgments}

This work was partially supported by CNPq/MCT, Brazil. GFH thanks FAPEMIG/MG, Brazil, for financial support. APCM thanks FAPERJ for partial support.

[1] Unruh W G and Zurek W H 1989 Phys. Rev. D 401071

[2] Hu B L, Paz J P and Zhang Y 1992 Phys. Rev. D 452843

[3] Ullersma P 1966 Physica 32 56; 74; 90

[4] Haake F and Reibold R 1982 Phys. Rev. A 322462

[5] Caldeira A O and Leggett A 1983 J Ann. Phys. 149374

[6] Grabert H, Schramm P and Ingold, G-L 1988 Phys. Rep. 168115

[7] Andion N P, Malbouisson A P C and Mattos Neto A 2001 J. Phys. A: Math. Gen. 343735

[8] Flores-Hidalgo G, Malbouisson A P C and Milla Y W 2002 Phys. Rev. A 65063414

[9] Malbouisson A P C 2003 Ann. Phys. 308373

[10] Flores-Hidalgo G and Malbouisson A P C 2002 Phys. Rev. A 66042118

[11] Flores-Hidalgo G and Malbouisson A P C 2005 Phys. Lett. A 33737

[12] Flores-Hidalgo G and Milla Y W 2005 J. Phys. A: Math. Gen. 387527

[13] Casana R, Flores-Hidalgo G and Pimentel B M 2005 Phys. Lett. A 3371

[14] Polonsky N 1964 Doctoral thesis (Paris: Ecole Normale Supérieure)

[15] Haroche S 1964 Doctoral thesis (Paris: Ecole Normale Supérieure)

[16] Petrosky T, Ordonez G and Prigogine I 2003 Phys. Rev. A 68022107

[17] Ford G W, Lewis JT and O'Connell R F 1996 Ann. Phys. 252362

[18] Audoin C, Cohen-Tannoudji C, Haroche S and Schermann J P 1970 Phys. Rev. Lett. 24861

[19] Cohen-Tannoudji C and Haroche S 1970 Phys. Rev. Lett. 24974

[20] Haroche S and Liberman S 1977 Frontiers in Laser Spectroscopy, R. Balian (ed) (Amsterdam: North-Holland)

[21] Haroche S, Brune M and Raimond J M 1991 Europhys. Lett. 1419

[22] Likhoded A K and Pronko G P 1997 Int. Journ. Theor. Phys. 362335 
[23] Thirring W and Schwabl F 1964 Ergeb. Exakt. Naturw. 36219 